\title{
IV Congresso Brasileiro de Nutrição Oncológica do INCA/VII Jornada Internacional de Nutrição Oncológica/VII Jornada Luso- -Brasileira em Nutrição Oncológica
}

Local: Centro de Convenções - Royal Tulip Hotel Rua Aquarela do Brasil, 75 - São Conrado. Rio de Janeiro (RJ), Brasil

Data: 29 e 30 de Outubro de 2015 


\title{
Triagem Nutricional, Sintomas Gastrointestinais e Capacidade Funcional em Idosos com Câncer Avançado \\ Nutritional Screening, Gastrointestinal Symptoms and Functional Capacity in Elderly Patients with Advanced Cancer \\ Evaluación Nutricional, Síntomas Gastrointestinales y Capacidad Funcional en Personas Mayores con Cáncer Avanzado
}

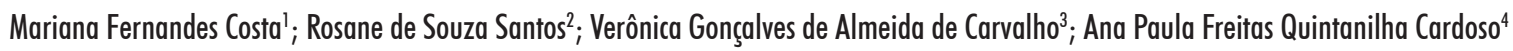

\section{Resumo}

Introdução: A Mini Avaliação Nutricional é um instrumento de triagem nutricional para idosos, sendo a perda da capacidade funcional e a ocorrência de sintomas gastrointestinais frequentes no câncer avançado. Objetivo: Avaliar o estado nutricional de idosos com câncer avançado e sua associaçáo com sintomas gastrointestinais e capacidade funcional. Método: Estudo observacional realizado com pacientes de ambos os sexos, acima de 65 anos, internados em Unidade de Cuidados Paliativos oncológicos, em novembro de 2014. O estado nutricional foi avaliado através da Mini Avaliação Nutricional e a capacidade funcional, pelo Karnofsky Perfomance Status. Análise estatística realizada pelos testes Qui-quadrado, Anova e regressão logística no Stata 12.0. Estudo aprovado pelo Comitê de Ética em Pesquisa do Instituto Nacional do Câncer José Alencar Gomes da Silva (688.258). Resultados: Amostra de 40 pacientes, média de $73( \pm 5,1)$ anos, sendo 55\% mulheres e 60\% de indivíduos com Karnofsky Perfomance Status de 30\%. Pela triagem nutricional, $77,5 \%$ desnutridos e 22,5\% em risco de desnutrição; $62,5 \%$ com diminuição severa da ingestão alimentar; 65\% com perda de peso maior que $3 \mathrm{Kg}$ em 3 meses; 47,5\% náo deambulavam; 27,5\% com demência ou depressáo grave; $42,5 \%$ com circunferência de panturrilha menor que $31 \mathrm{~cm}$. Sintomas mais frequentes: hiporexia (45\%), disfagia (35\%), náuseas (30\%), vômitos (20\%), xerostomia (20\%) e plenitude gástrica (10\%). A Mini Avaliação Nutricional apresentou associação estatisticamente significativa $(\mathrm{p}=0,05)$ com Karnofsky Perfomance Status. Apenas o vômito foi estatisticamente associado à desnutrição $(\mathrm{p}=0,05)$. Conclusão: Todos apresentaram desnutrição ou risco nutricional, associado à baixa capacidade funcional e vômitos, ressaltando a importância da triagem nutricional para intervenção. Palavras-chave: Avaliação Nutricional; Sinais e Sintomas; Idoso; Neoplasias

\section{Declaraçáo de Conflito de Interesses: Nada a Declarar.}

\footnotetext{
${ }^{1}$ Nutricionista-clínica. Doutora em Alimentação, Nutrição e Saúde da Universidade do Estado do Rio de Janeiro (UERJ). Doutora em Psicologia Social da UERJ. Serviço de Nutrição do Hospital do Câncer IV (HC IV). Instituto Nacional de Câncer José Alencar Gomes da Silva (INCA). Rio de janeiro (RJ), Brasil.E-mail: marifcosta@gmail.com.

${ }^{2}$ Nutricionista-clínica. Mestre em Neurologia da Universidade Federal do Estado do Rio de Janeiro (UNIRIO). Serviço de Nutrição do HC IV/INCA. Rio de janeiro (RJ), Brasil.E-mail: rosanerss@yahoo.com.br.

${ }^{3}$ Nutricionista-clínica. Especialização em Nutrição Funcional pela Universidade Ibirapuera. Especialização em Nutrição Esportiva pela Universidade Veiga de Almeida (UVA). Serviço de Nutriçăo do HC IV/INCA. Rio de janeiro (RJ), Brasil. E-mail: veroniq@terra.com.br.

${ }^{4}$ Nutricionista-clínica. Especialização em Nutrição Oncológica do INCA. Especialização em Nutrição Clínica pela UVA. Serviço de Nutrição do HC IV/ INCA. E-mail: anafqc@hotmail.com - Niterói (RJ), Brasil.

Endereço para correspondência: Mariana Fernandes Costa. Rua Domingues de Sá 381/704 - Icaraí. Niterói (RJ), Brasil. CEP: 24220-090. E-mail: marifcosta@gmail.com.
} 


\title{
Análise da Sobrevida de Pacientes Idosos com Câncer segundo Parâmetros Nutricionais
}

Survival Analysis of Elderly Cancer Patients according Nutritional Parameters Análisis de Supervivencia de los Pacientes Ancianos con Cáncer según los Parámetros Nutricionales

\author{
Mariana Vieira Barbosa ; Nivaldo Barroso de Pinhoㄹ; Viviane Dias Rodrigues ${ }^{3}$ Anke Bergmann ${ }^{4}$; Luiz Claudio Santos Thuler ${ }^{5}$ Renata Brum Martucci ${ }^{6}$
}

\section{Resumo}

Introdução: A desnutrição no paciente idoso associa-se com redução da sobrevida, hospitalização prolongada, desfecho desfavorável e reduçáo da funcionalidade. Objetivo: Analisar a sobrevida de idosos com câncer de acordo com os parâmetros nutricionais avaliados. Método: Estudo de coorte com pacientes idosos acima de 65 anos admitidos no Instituto Nacional de Câncer José Alencar Gomes da Silva, entre julho e novembro de 2014. Foram coletados: idade, gênero, Mini Avaliação Nutricional Versão Reduzida (MNA-VR), peso, altura, Índice de Massa Corporal (IMC), Circunferência da Panturrilha (CP) e tempo de sobrevida até seis meses pelo método de Kaplan-Meier, e comparação das curvas pelo teste de log-rank e regressão de Cox. Resultados: Participaram do estudo 143 idosos, com média de idade de $72,9 \pm 6,5$ anos, $54 \%$ eram homens e $40 \%$ tinham tumores no sistema digestivo. A média de peso foi $64,8 \pm 14,4 \mathrm{~kg}$, IMC $24,8 \pm 4,6 \mathrm{~kg} / \mathrm{m}^{2}$ e CP $33 \pm 4,2 \mathrm{~cm}$. Segundo a MNA-VR, $31 \%$ eram desnutridos, $41 \%$ estavam sob risco de desnutriçáo e $28 \%$ eram normais (escore médio de $8,9 \pm 3,2$ ). A sobrevida média foi de 3,9 2 2,0 meses, ocorrendo 34 óbitos (24\%). Desnutrição, $\mathrm{CP}<31 \mathrm{~cm}$ e motivo da internação foram considerados fatores independentes associados ao óbito. A sobrevida foi menor nos desnutridos pela MAN-VR ( $<<0,001)$ e $\operatorname{com} \mathrm{CP}<31 \mathrm{~cm}$ ( $\mathrm{p}=0,001)$. Não houve diferença quando os pacientes foram estratificados segundo IMC. O modelo de Cox mostrou que ser desnutrido aumentou o risco de morte (HR 6,6; IC 95\%:1,4-31; p=0,016), quando ajustado pela CP, motivo da internação e idade. Conclusáo: Dos parâmetros estudados, a classificação de desnutrição da MAN-VR foi o fator mais associado com a redução na sobrevida de idosos com câncer.

Palavras-chave: Sobrevida; Idoso; Neoplasias; Avaliação nutricional; Desnutrição

\section{Declaraçáo de Conflito de Interesses: Nada a Declarar.}

\footnotetext{
${ }^{1}$ Nutricionista. Especialista em Nutrição Oncológica pelo Instituto Nacional de Câncer José Alencar Gomes da Silva (INCA). Especializanda em Pesquisa Pós-residência da Pós-Graduação em Oncologia do INCA. Rio de Janeiro (RJ), Brasil. E-mail: marivieira89@gmail.com.

${ }^{2}$ Nutricionista. Mestre em Nutrição Humana pela Universidade Federal do Rio de Janeiro (UFRJ). Coordenador da Divisão de Apoio Técnico do Hospital do Câncer I (HC I)/INCA. E-mail: npinho@inca.gov.br.

${ }^{3}$ Nutricionista. Mestre em Ciências Médicas pela Universidade do Estado do Rio de Janeiro (UERJ). Chefe do Serviço de Nutrição e Dietética do HC I/INCA. E-mail: viviane.rodrigues@inca.gov.br.

${ }^{4}$ Fisioterapeuta. Doutora em Saúde Pública pela Fundação Oswaldo Cruz (Fiocruz). Pesquisadora da Divisão de Pesquisa Clínica e Incorporaçăo Tecnológica do INCA. Professora-adjunta do Centro Universitário Augusto Motta (UNISUAM). Rio de Janeiro (RJ), Brasil. E-mail: abergmann@inca.gov.br.

${ }^{5}$ Médico. Doutor em Medicina pela UFRJ. Pesquisador da Divisão de Pesquisa Clínica e Incorporaçấo Tecnológica do INCA. Professor-associado da Universidade Federal do Estado do Rio de Janeiro (UNIRIO). Rio de Janeiro (RJ), Brasil. E-mail: lthuler@inca.gov.br.

${ }^{6}$ Nutricionista. Doutora em Ciências pela UFRJ. Nutricionista do Serviço de Nutrição e Dietética do HC I/INCA. Professora-adjunta da UERJ. Rio de Janeiro (RJ), Brasil. E-mail: renata.martucci@inca.gov.br.

Endereço para correspondência: Mariana Vieira Barbosa. Rua Benjamin Constant, 134/409 - Glória. Rio de Janeiro (RJ), Brasil. CEP: 20.241-150. E-mail: marivieira89@gmail.com.
} 


\section{Adaptação Transcultural de um Questionário de Triagem Nutricional em Pacientes Pediátricos Hospitalizados com Câncer Cross-Cultural Adaptation of a Questionnaire of Nutritional Screening in Pediatric Patients Hospitalized with Cancer Adaptación Transcultural de un Cuestionario de Triaje Nutricional en Pacientes Pediátricos Hospitalizados con Cáncer}

Danúbia da Cunha Antunes Saraiva ${ }^{1}$; Wanélia Vieira Afonso ${ }^{2}$; Nivaldo Barroso de Pinho ${ }^{3}$; Wilza Arantes Ferreira Peres ${ }^{4}$ Patricia de Carvalho Padilha ${ }^{5}$

\section{Resumo}

Introdução: A assistência nutricional a pacientes pediátricos com câncer deve garantir a identificação precoce do risco nutricional. Em 2007, Secker e Jeejeebhoy desenvolveram a ferramenta Pediatric Subjective Global Nutritional Assessment; e, em 2012, publicaram o instrumento sugerido, que utiliza informaçóes recentes sobre antropometria (peso e estatura), ingestão dietética, frequência e duração dos sintomas gastrointestinais, estresse metabólico, capacidade funcional e exame físico do paciente pediátrico. Objetivo: Realizar a adaptação transcultural da Pediatric Subjective Global Nutritional Assessment, citada aqui como Avaliação Nutricional Subjetiva Global Pediátrica (ANSGP), para o português brasileiro para utilização em crianças e adolescentes hospitalizados com câncer. Método: $\mathrm{O}$ processo de adaptação transcultural seguiu as seguintes etapas: equivalência conceitual, de item, semântica, operacional e validação do conteúdo. No processo de adaptação transcultural, o questionário foi discutido com um grupo de especialistas da área de nutrição e pediatria, e pré-testado em 32 pacientes pediátricos da enfermaria de oncopediatria e hematologia pediátrica de um Centro de Referência em Oncologia, entre 2 a 18 anos. Resultados: A versão traduzida da ANSGP necessitou de poucos ajustes para atingir todas as equivalências propostas e alcançou sua validaçáo do conteúdo, permitindo uma versão com linguagem acessível e compreensão por parte da população-alvo. Todas as etapas fundamentaram a adaptação transcultural do instrumento a ser utilizado e compreendido em nosso meio. Conclusáo: Nesse processo, foi obtida uma versão final do instrumento adaptado culturalmente, sendo este o primeiro passo para disponibilizar uma ferramenta de triagem do estado nutricional de pacientes com câncer na faixa etária pediátrica.

Palavras-chave: Triagem; Pediatria; Neoplasias; Comparaçáo Transcultural

\section{Declaraçáo de Conflito de Interesses: Nada a Declarar.}

\footnotetext{
${ }^{1}$ Nutricionista. Mestre em Nutrição Clínica pela Universidade Federal do Rio de Janeiro (UFRJ). Nutricionista do Serviço de Nutrição e Dietética do Hospital do Câncer I (HC I) do Instituto Nacional de Câncer José Alencar Gomes da Silva (INCA). Rio de Janeiro (RJ), Brasil. E-mail: dcasaraiva@gmail.com.

${ }^{2}$ Nutricionista. Especialização em Nutrição Clínica em Pediatria pela Faculdade de Medicina da Universidade de São Paulo (USP). Mestranda em Nutrição Clínica pela UFRJ. Nutricionista do Serviço de Nutriçăo e Dietética do HC I/INCA. Rio de Janeiro (RJ), Brasil. E-mail: nutri.wanelia@uol.com.br.

${ }^{3}$ Nutricionista. Mestre em Nutrição Humana pela UFRJ. Doutorando em Ciências Nutricionais pela UFRJ. Coordenador da Divisão de Apoio Técnico do HCI/INCA. E-mail: npinho@inca.gov.br

${ }^{4}$ Nutricionista. Doutora em Clínica Médica pela UFRJ. Professora-adjunta do Instituto de Nutrição Josué de Castro da UFRJ. Rio de Janeiro (RJ), Brasil. E-mail:wilza@nutricao.ufrj.br.

${ }^{5}$ Nutricionista. Doutora em Ciências Nutricionais pela UFRJ. Professora-adjunta do Instituto de Nutrição Josué de Castro da UFRJ. Rio de Janeiro (RJ), Brasil.E-mail: paticpadilha@yahoo.com.br.

Endereço para correspondência: Serviço de Nutrição e Dietética. Danúbia da Cunha Antunes Saraiva. Praça Cruz Vermelha, 23/5o andar - Centro. Rio de Janeiro (RJ), Brasil. CEP: 20230-130. E-mail: dcasaraiva@gmail.com.
} 


\title{
Avaliação do Estado Nutricional e Prognóstico Inflamatório Nutricional em Pacientes Portadores de Carcinoma Gastrointestinal em Belém (PA), Brasil
}

Nutritional Assessment and Prognostic Inflammatory Nutritional Index in Patients with Gastrointestinal Carcinoma in Belém (PA), Brazil

Evaluación del Estado Nutricional y El Pronóstico Inflamatorio Nutricional en Pacientes con Cáncer Gastrointestinal en Belém (PA), Brasil

\author{
Tayana Vago de Miranda ${ }^{1}$; Marília de Souza Araújo² ${ }^{2}$ Liliane Maria Messias Machado ${ }^{3}$; Jamille Jheniffer Nascimento Farias ${ }^{4}$; \\ Joyce de Nazaré Monteiro dos Santos ${ }^{5}$
}

\section{Resumo}

Introduçáo: A avaliação do estado nutricional e a inflamação sistêmica em pacientes oncológicos permitem a identificação de indivíduos em risco de complicações clínicas associadas ao comprometimento nutricional, otimizando a intervenção precoce e melhores resultados clínicos. Objetivo: Descrever o estado nutricional e o índice de prognóstico inflamatório nutricional (IPIN) em pacientes com carcinoma gastrointestinal. Método: Estudo transversal, descritivo, observacional, com indivíduos diagnosticados com carcinoma gastrointestinal, atendidos no Hospital Universitário João de Barros Barreto (HUJBB) em Belém-PA. Utilizaram-se, na avaliação nutricional, o Índice de Massa Corporal (IMC), Circunferência do braço (CB), Circunferência Muscular do Braço (CMB), Área Muscular do Braço Corrigida $(\mathrm{AMBc})$, Prega Cutânea Triciptal (PCT) e Avaliação Subjetiva Global Produzida pelo Paciente (ASG-PPP). O IPIN foi obtido por meio da relação PCR/albumina. Resultados: Foram avaliados 44 pacientes, sendo 63,30\% do sexo masculino, com idade média de 61,2 anos $( \pm 13,3)$, diagnosticados com neoplasia de estômago $(63,60 \%)$. Verificaram-se $20,50 \%$ de eutrofia para adultos e 42,30\% para idosos, no IMC. Obtiveram-se 59,10\% de depleção grave na AMBc e 54,50\% por meio da PCT, e 75,00\% com presença de depleção em algum grau em CB e 68,18\% em CMB. Na ASG-PPP, identificaram-se desnutrição grave em $63,60 \%$ dos pacientes e no IPIN, presença de risco em 93,18\% dos pacientes, com maior prevalência para médio risco de complicação (56,80\%). Não se obteve correlaçáo estatística entre marcadores do estado nutricional e IPIN. Conclusão: Os resultados evidenciam alta prevalência de comprometimento nutricional e presença de risco de complicaçóes, reforçando a importância de acompanhamento desses parâmetros na assistência nutricional de pacientes oncológicos.

Palavras-chave: Estado Nutricional; Inflamação; Neoplasias Gastrointestinais

\section{Declaraçáo de Conflito de Interesses: Nada a Declarar.}

\footnotetext{
1. Nutricionista. Doutoranda do Programa de Pós-Graduação em Oncologia e Ciências Médicas da Universidade Federal do Pará (UFPA). Belém (PA), Brasil. E-mail: tayana.vdm@gmail.com.

2. Nutricionista. Doutora em Ciências da Saúde pela Universidade de Brasília. Professora-associada da Faculdade de Nutrição da UFPA. Belém (PA), Brasil.E-mail:maraujo@ufpa.br.

3. Nutricionista. Doutora em Ciências da Saúde pela Universidade de Brasília. Professora-adjunta I da Faculdade de Nutrição da UFPA. Belém (PA), Brasil. E-mail: liliane.machado25@gmail.com.

4. Graduanda da Faculdade de Nutrição da UFPA. Belém (PA), Brasil. E-mail: jamille.nf@hotmail.com.

5. Graduanda da Faculdade de Nutrição da UFPA. Belém (PA), Brasil. E-mail: Joyce_nutri92@hotmail.com.

Endereço para correspondência: Tayana Vago de Miranda. Rua Fernando Guilhon, Pass. União, 481 - Cremação. Belém (PA), Brasil. CEP: 66045-550.

E-mail: tayana.vdm@gmail.com.
} 


\section{Albumina Sérica e Contagem Total de Linfócitos como Marcadores Prognósticos em Pacientes com Câncer \\ Serum Albumin and Total Lymphocyte Count as Prognostic Markers in Cancer Patients

\author{
Albúmina Sérica y la Cifra de Linfocitos Totales como Marcadores Pronósticos \\ en Pacientes con Cáncer
}

Uiara Mara Soares Nunes de Souza ; Roberta Sônia Rodrigues Álvares²; Edson Misael Guimarães ${ }^{3}$ Maria Gonçalves Soares ${ }^{4}$

\section{Resumo}

Introdução: Estudos vêm sendo desenvolvidos para encontrar marcadores de permanência hospitalar e mortalidade em pacientes hospitalizados. A albumina sérica e a Contagem Total de Linfócitos (CTL) têm sido correlacionadas com o estado nutricional e a gravidade da doença. Objetivo: Avaliar a relação da albumina sérica e linfocitometria com a permanência hospitalar e mortalidade no paciente oncológico. Método: Estudo transversal. Foram avaliados 61 pacientes (60,6\% sexo feminino) atendidos na enfermaria oncológica do Hospital Santa Casa de Belo Horizonte, voluntários, com idade média de $51,5( \pm 18,3)$ anos. Pesquisa Variável. A coleta dos dados referentes à albumina, e à linfocitometria foi realizada no sistema eletrônico de solicitação de exames. A interpretação dos resultados tanto de CTL (\% linfócitos x leucócitos (ml)/100) como da albumina seguiu o esquema proposto por Blackburn. Resultados: Com relação ao desfecho clínico, 27,9\% evoluíram para óbito. Além disso, 96,7\% da população apresentaram alterações leucocitárias, sendo que 27,9\% tiveram CTL considerada grave. A média da CTL foi de 1.933,04 $\pm 3.271,74$ leucócitos e de albumina foi de 2,40 $\pm 0,63 \mathrm{mg} / \mathrm{dL}$. Entre as variáveis analisadas, somente a albumina sérica $(\mathrm{p}=0,002)$ foi significativa para a associação com o desfecho alta ou óbito. Conclusáo: Conclui-se pelo estudo que o nível sérico da albumina pode ser utilizado como marcador de desfecho clínico (alta e óbito) no paciente oncológico, enquanto a CTL não foi um bom marcador.

Palavras-chave: Albumina; Linfócitos; Prognóstico; Mortalidade; Oncologia

\section{Declaraçáo de Conflito de Interesses: Nada a Declarar.}

\footnotetext{
${ }^{1}$ Nutricionista. Especialista em Gestão Gastronômica e Hoteleira. Especialista em Nutriçâo Hospitalar. Coordenadora da Nutrição Clinica do Hospital Santa Casa de Belo Horizonte. Vice-presidente da Equipe Multiprofissional de Terapia Nutricional (EMTN) do Hospital Santa Casa de Belo Horizonte. Belo Horizonte (MG), Brasil.E-mail: umsn_souza@yahoo.com.br.

${ }^{2}$ Nutricionista. Mestre em Bioquímica Metabólica e Fisiológica. Especialista em Terapia Nutricional e Nutrição Clínica. E-mail: robertarodrigues21@yahoo.com.br.

${ }^{3}$ Enfermeiro. Mestre em Ciências da Saúde - Epidemiologia. Professor-adjunto da Fundaçáo Comunitária de Ensino Superior de Itabira. E-mail: edilsonenf@gmail.com.

${ }^{4}$ Nutricionista. Especialista em Terapia Nutricional e Nutrição Clínica. Gerente da Unidade de Nutrição do Hospital Maria Amélia Lins - Fhemig. E-mail: marianut@yahoo.com.br.

Enderę̧o para correspondência: Uiara Mara Soares Nunes de Souza. Rua Tenente Garro, 101, apartamento 01 - Santa Efigênia. Belo Horizonte (MG), Brasil. CEP: 30240-360.E-mail: umsn_souza@yahoo.com.br.
} 


\title{
Comparação entre Dois Instrumentos de Identificação de Risco Nutricional em Pacientes Idosos em Tratamento Oncológico no Instituto do Câncer do Estado de São Paulo
} Comparing Two Instruments of Identification in Nutritional Risks among Elderly Patients through Oncology Treatment at Cancer Institute of the State of São Paulo Comparación entre dos Instrumentos de Identificación de Riesgo Nutricional en Pacientes Mayores en Tratamiento Oncológico en el Instituto de Cáncer del Estado de São Paulo

Marcella Esbrogeo Call'; Gislaine Aparecida Ozorioº ; Juliana Bravo Guerrero ${ }^{3}$; Maria Manuela Ferreira Almeida ${ }^{4}$, Serena Nunes Vianna ${ }^{5}$

\begin{abstract}
Resumo
Introduçáo: $\mathrm{O}$ câncer está associado ao envelhecimento e afeta diretamente o estado nutricional, sendo essencial um instrumento de triagem nutricional (TN) que identifique o risco nutricional. Objetivos: Avaliar a sensibilidade e especificidade entre dois instrumentos de TN em pacientes idosos oncológicos. Método: Estudo transversal prospectivo com pacientes idosos (acima de 65 anos), portadores de tumores malignos de ambos os sexos, internados no Instituto do Câncer do Estado de São Paulo (ICESP), de setembro a novembro de 2014. Foram triados pelos dois instrumentos de TN: (Mini Nutritional Assessment - Short Form (MNA-SF) e Nutritional Risk Screening (NRS 2002), em até 24 horas após internação. A medida da Circunferência da Panturrilha (CP) foi utilizada como padrão-ouro e foram calculadas as medidas de desempenho diagnóstico (sensibilidade, especificidade e acurácia). As análises estatísticas foram feitas com o programa Stata 11.1. Resultados: Foram avaliados 286 pacientes idosos, sendo 172 (60,1\%) do sexo masculino e $114(39,9 \%)$ do sexo feminino, com idade média 72,4 \pm 4,6 anos, e o tempo de internação 5,1 \pm 3,6 dias. Foram classificados com risco nutricional pela MNA-SF 209 pacientes (73,1\%), e pela NRS-2002 109 pacientes (38,1\%), com uma diferença estatística de $\mathrm{p}<0,001$. As medidas de desempenho diagnóstico foram: sensibilidade $96,6 \%$, especificidade $98,7 \%$ e acurácia $72 \%$ para MNA-SF; e sensibilidade $65,6 \%$, especificidade $40,1 \%$ e acurácia $45,8 \%$ para NRS-2002. Conclusão: Dos instrumentos de triagem nutricional avaliados, a MNA-SF apresentou maior sensibilidade, especificidade e acurácia em comparação a NRS-2002 em pacientes idosos oncológicos, segundo este estudo.
\end{abstract}

Palavras-chave: Câncer; Idoso; Estado Nutricional; Sensibilidade; Especificidade

\section{Declaração de Conflito de Interesses: Nada a Declarar.}

\footnotetext{
${ }^{1}$ Nutricionista. Aprimoramento em Nutrição em Hematologia e Oncologia. Nutricionista do Instituto do Câncer do Estado de São Paulo (ICESP). São Paulo (SP), Brasil. E-mail: marcella.esbrogeo@hc.fm.usp.br.

${ }^{2}$ Nutricionista. Mestranda em Gastroenterologia. Coordenadora Administrativa da Equipe Multiprofissional de Terapia Nutricional (EMTN) do ICESP. São Paulo (SP), Brasil. E-mail: gislaine.ozorio@hc.fm.usp.br.

${ }^{3}$ Nutricionista. Especialização em Nutrição Clínica no Sistema Digestório. Nutricionista do ICESP. São Paulo (SP), Brasil. E-mail: juliana.guerrero@hc.fm.usp.br.

${ }^{4}$ Nutricionista. Especialização em Gestão da Política de Alimentação e Nutrição. Coordenadora de Nutrição Clínica do ICESP. São Paulo (SP), Brasil. E-mail: manuela.alves@hc.fm.usp.br.

${ }^{5}$ Nutricionista. MBA Executivo em Saúde: Ênfase em Gestão de Clínicas e Hospitais. Gerente do Serviço de Nutrição do ICESP. São Paulo (SP), Brasil. E-mail: serena.vianna@hc.fm.usp.br.

Endereço para correspondência: Marcella Esbrogeo. Rua Oscar Freire, 1.967, apartamento 54A - Bloco 1. Pinheiros. São Paulo (SP), Brasil. CEP: 05409011.E-mail: marcella.esbrogeo@hc.fm.usp.br.
} 


\title{
Correlação entre o Ângulo de Fase e Indicador Funcional e de Estado Nutricional em Pacientes Idosos com Diagnóstico de Neoplasia na Região Torácica Correlation between Phase Angle, Functional Indicator and Nutritional Status in Elderly Patients with Diagnosis of Neoplasia in the Chest Region Correlación entre Ángulo de Fase, Indicador Funcional y Estado Nutricional en Pacientes Mayores con Diagnóstico de Neoplasia en la Región de Pecho
}

\author{
Otávio Rocha Esperança ${ }^{1}$; Ana Carolina Alves de Lima ${ }^{2}$; Anne Caroline Terra Luquetti ${ }^{3}$ Emilia Delesderrier Franco ${ }^{4}$; Jessica Rodrigues Lopes ${ }^{5}$; \\ Daiane Spitz de Souza ${ }^{6}$
}

\section{Resumo}

Introdução: $\mathrm{O}$ estado nutricional influencia diretamente no prognóstico de pacientes oncológicos idosos, sendo o ângulo de fase (AF) uma medida simples de ser realizada que pode facilitar a triagem e a definição de conduta nutricional nesses pacientes. Objetivo: Evidenciar a correlaçáo do AF com indicadores de estado nutricional e funcional, usados na avaliação de pacientes idosos internados em um Hospital Universitário entre 2014 e 2015 com diagnóstico de neoplasia na região torácica. Método: Estudo transversal com 28 pacientes com diagnóstico de neoplasia de esôfago ou pulmão em pré-operatório. Os indicadores analisados foram: Análise Subjetiva Global Produzida Pelo Próprio Paciente (ASG-PPP), Karnofsky Performance Status (KPS), Índice de Quételet (IMC), Percentual de Perda de Peso na Internação (\%PP), Dobra Cutânea Triciptal (DCT), Circunferência Muscular do Braço (CMB), AF, Ângulo de Fase Padrão (AFP). Para análise estatística, foi realizada a Correlação de Spearman(p) no software SPSS versão 20.0. Resultados: A média de idade foi 70 anos; de IMC=24,46; ASG-PPP=9; $\% \mathrm{PP}=8$; a mediana do KPS foi 90 . O AF se correlacionou positivamente com: IMC ( $p=0,390 ; p=0,04)$, KPS ( $p=0,473 ; p=0,011)$ e CMB ( $p=0,656 ; p=0,000)$ e negativamente com a ASG-PP $(p=-0,391 ; p=0,4)$. Conclusão: $O$ AF apresentou correlação positiva com indicador de avaliação funcional e de estado nutricional, nesta amostra, sugerindo sua utilização como ferramenta de avaliação nutricional, subsidiando a prática clínica.

Palavras-chave: Impedância Bioelétrica; Estado Nutricional; Prognóstico; Neoplasias

\section{Declaraçáo de Conflito de Interesses: Nada a Declarar.}

\footnotetext{
Divisão de Nutrição do Hospital Universitário Pedro Ernesto. Universidade do Estado do Rio de Janeiro (HUPE/UERJ).

${ }^{1}$ Nutricionista. Residente de Nutrição Clínica. Divisão de Nutrição do HUPE/UERJ. Rio de Janeiro (RJ), Brasil. E-mail: irouma@gmail.com.

${ }^{2}$ Nutricionista. Residente de Nutrição Clínica. Divisão de Nutrição do HUPE/UERJ. Rio de Janeiro (RJ), Brasil. E-mail: anacarol.lima2@gmail.com.

${ }^{3}$ Nutricionista. Residente de Nutrição Clínica. Divisāo de Nutrição do HUPE/UERJ. Rio de Janeiro (RJ), Brasil. E-mail: anne.luquetti@gmail.com.

${ }^{4}$ Nutricionista. Residente de Nutrição Clínica. Divisão de Nutrição do HUPE/UERJ. Rio de Janeiro (RJ), Brasil. E-mail: emiliadeles@gmail.com.

${ }^{5}$ Nutricionista. Residente de Nutrição Clínica. Divisão de Nutrição do HUPE/UERJ. Rio de Janeiro (RJ), Brasil. E-mail: jessicalopes.nutri@gmail.com.

${ }^{6}$ Nutricionista. Mestre em Nutriçâo Humana pela Universidade Federal do Rio de Janeiro (UFRJ). Pós-graduada em Nutriçăo Oncológica do Instituto

Nacional de Câncer José Alencar Gomes da Silva (INCA). Pós-graduada em Nutrição Clínica pela UFRJ. Centro Universitário de Controle do Câncer do HUPE/UERJ. Rio de Janeiro (RJ), Brasil. E-mail: daianespitz@hotmail.com.

Endereço para correspondência: Otávio Rocha Esperança. Estrada do Pau Ferro 451, Jacarepaguá. Rio de Janeiro (RJ), Brasil. CEP: 22743-050 E-mail: 1irouma@gmail.com.
} 